\title{
Article \\ Dichroic Circular Polarizers Based on Plasmonics for Polarization Imaging Applications
}

\author{
Junyan Zheng ${ }^{1,+}$, Xin He ${ }^{1,2, *,+}$, Paul Beckett ${ }^{3}$, Xinjie Sun ${ }^{1}$, Zixin Cai ${ }^{1}$, Wenyi Zhang ${ }^{1}$, Xu Liu $^{1}$ \\ and Xiang Hao $1,2, *$ \\ 1 State Key Laboratory of Modern Optical Instrumentation, College of Optical Science and Technology, \\ Zhejiang University, Hangzhou 310027, China; junyan_zheng@zju.edu.cn (J.Z.); sunxj@zju.edu.cn (X.S.); \\ czx805660580@zju.edu.cn (Z.C.); 21930064@zju.edu.cn (W.Z.); liuxu@zju.edu.cn (X.L.) \\ 2 Intelligent Optics \& Photonics Research Center, Jiaxing Research Institute, Zhejiang University, \\ Jiaxing 314000, China \\ 3 School of Engineering, RMIT University, Melbourne, VIC 3000, Australia; paul.beckett@rmit.edu.au \\ * Correspondence: xinhe.wins@outlook.com (X.H.); haox@zju.edu.cn (X.H.) \\ + These authors contribute equally to this work.
}

Citation: Zheng, J.; He, X.; Beckett, P.; Sun, X.; Cai, Z.; Zhang, W.; Liu, X.; Hao, X. Dichroic Circular Polarizers Based on Plasmonics for Polarization Imaging Applications. Nanomaterials 2021, 11, 2145. https://doi.org/ $10.3390 /$ nano11082145

Academic Editors: Takashi Jin and Horacio Cabral

Received: 16 July 2021

Accepted: 18 August 2021

Published: 23 August 2021

Publisher's Note: MDPI stays neutral with regard to jurisdictional claims in published maps and institutional affiliations.

Copyright: (c) 2021 by the authors. Licensee MDPI, Basel, Switzerland. This article is an open access article distributed under the terms and conditions of the Creative Commons Attribution (CC BY) license (https:// creativecommons.org/licenses/by/ $4.0 /)$.

\begin{abstract}
Dichroic circular polarizers (DCP) represent an important group of optical filters that transfer only that part of the incident light with the desired polarization state and absorb the remainder. However, DCPs are usually bulky and exhibit significant optical loss. Moreover, the integration of these kinds of DCP devices can be difficult and costly as different compositions of chemicals are needed to achieve the desired polarization status. Circular polarizers based on metasurfaces require only thin films in the order of hundreds of nanometers but are limited by their sensitivity to angle of incidence. Furthermore, few existing solutions offer broadband operation in the visible range. By using computational simulations, this paper proposes and analyses a plasmonic DCP structure operating in the visible, from $400 \mathrm{~nm}$ to $700 \mathrm{~nm}$ which overcomes these drawbacks. The resulting circular dichroism transmission (CDT) is more than 0.9 , and the maximum transmission efficiency is greater than $78 \%$ at visible wavelengths. These CDT characteristics are largely independent of angle of incidence up to angles of 80 degrees.
\end{abstract}

Keywords: metasurface; circular polarizer; polarization imaging

\section{Introduction}

Unlike amplitude (brightness), wavelength (color), and other light information that the human eye is able to detect without using additional optical devices, polarization information cannot be directly seen. At the same time, circular polarizers are critical optical devices in a broad range of polarized imaging applications such as the classification of biological molecules [1], medical analysis [2], chemical identification [3], quantum information [4], and many other research fields.

Dichroic circular polarizers (DCPs) are an important class of polarizers that attract increasing attention. Their key advantage over other circular polarizers is their ability to absorb the light components with unwanted polarization states instead of reflecting them, thereby reducing interference and other effects on the incident light [5,6]. However, DCPs are usually bulky and can have significant optical loss [6-10]. For example, commercial DCP devices from [9] exhibit transmission efficiencies of around $42 \%$ due to the strong absorption of their polymer-based materials. Similarly, while the transmission efficiency of the circular polarizers offered by [10] can reach over $80 \%$, the relative circular dichroism transmission (CDT) is only 0.33 in the visible band. Moreover, this type of circular polarizer needs to use a range of material compositions and/or chemical concentrations to achieve its desired optical properties (e.g., polarization angle and phase difference). Creating polarization sensitive cameras suited to imaging applications [11-13] based on these techniques requires 
advanced overlay methods to integrate the polarizers onto a photodetector array, which is potentially both complicated and costly.

Metasurface-based circular polarizers do not exhibit these drawbacks. Firstly, their component nanostructures are usually fabricated from dielectric or metallic films with thicknesses in the range of a few hundred nanometers and are therefore not bulky [14-17]. Moreover, the operating wavelengths can be simply tuned by varying the size, spacing, and/or period of the nanostructures [8,15-29]. Lastly, metasurface-based optical devices have already been described that have smaller optical loss than existing systems. For example, dielectric and plasmonic metallic nanostructures have been demonstrated in $[5,17]$ with transmission efficiencies of more than $80 \%$ and of around $60 \%$ in $[20,21,30]$. As a result, there has been much activity in recent decades exploring metasurface-based polarizers across both academic research and industrial product development.

Metallic nanogratings are polarization-sensitive nanostructures and, thus, can be used to create linear polarizers [31,32]. Their operating wavelength can be simply tuned by varying the grating period, and the transmission efficiency is directly related to the separation between each nanograting element. The further integration of nanogratingbased linear polarizers with different polarization angles [33] onto conventional image sensors has allowed the development of compact linear polarization cameras.

Dichroic linear polarizers (DLPs) based on metallic nanoparticles are also of interest as they behave in the same way as conventional DLPs in that they absorb, rather than reflect, unwanted polarization states [34-37]. Metallic nanostructures behave as perfect electric conductors with high reflectance, but their optical properties can be simply manipulated. Although much work has been published to demonstrate this basic idea, there have been few demonstrations of metasurface-based circular polarizers working within the visible spectrum. One example is the chiral, two-layer metasurface based on twisted nanorods, described theoretically in [38]. By rotating the second nanorod by 45 degrees, the whole polarizer achieves a maximum transmission difference $(\Delta \mathrm{T})$ between the LHP and RHP states of around $58 \%$ at $1650 \mathrm{~nm}$. It is also able to distinguish LHP light from RHP light within a broad near IR wavelength range from $1500 \mathrm{~nm}$ to $1750 \mathrm{~nm}$ by absorbing the RHP component. However, these larger transmission differences can only be achieved at limited wavelengths.

Stacked multi-layer nanorod array structures with tailored rotational twist were presented in [31] that have operational bandwidths in the visible range from around 500 to $750 \mathrm{~nm}$. However, this structure becomes a phase converter at wavelengths less than $500 \mathrm{~nm}$, swapping the polarization state of the incident light (LCP to RCP or vice versa) by changing its phase difference. Moreover, its constituent gold nanorod material is expensive and is not CMOS-compatible. Circular polarizers based on helical metasurfaces with a high extinction ratio were presented in [39-41]. As the metallic structure is asymmetric along the propagation direction, RHP and LHP states can be distinguished over a relatively wide range within and just above the mid-infrared region from around $3 \mu \mathrm{m}$ to $10 \mu \mathrm{m}[39,40]$. However, the types of 3D nanostructures required here are difficult to build using typical nanofabrication facilities such as electron-beam lithography or focused ion beam. An alternative might be a maskless 3-D nano-printing system such as Nanoscribe [42], but in this case the restricted accuracy and feature sizes of these systems (typically $>300 \mathrm{~nm}$ ) will impose limits on the operating range, which is inversely proportional to the size (width, length, etc.) of the nanostructures.

Double-helical metasurface-based nanostructures have been shown to have a broad operating range across the whole visible spectrum. However, these structures are also very difficult to fabricate using any recent nanofabrication technique [41]. Although directioncontrolled bifunctional metasurface Polarizers, formed by inserting nanoslits with two different thicknesses into a thick gold film, have been demonstrated [43] to exhibit wideband operation from $600 \mathrm{~nm}$ to $1000 \mathrm{~nm}$, their thick metal layer results in low transmission efficiency-around $8 \%$ in the experiments of [43]. A planar chiral metasurface comprising double-layered dielectric-metal-dielectric resonant structures in the shape of a gammadion 
has been shown [44] to offer high transmission efficiency, but only over a very narrow band at around $1100 \mathrm{~nm}$. The metasurface polarization camera of [45], based on $\mathrm{TiO}_{2}$ nanorods, is capable of simultaneously taking polarized and 3D images with one snapshot. The metasurface can diffract circularly polarized light with four phase differences into four directions. Although the camera is capable of taking full Stokes images, it has two main drawbacks. Firstly, as it operates by diffraction, it will work only at a single wavelength $(532 \mathrm{~nm})$, which is determined by the period of the nanostructures. Further, as the four images result from diffracted rays that are therefore not parallel, an aspheric lens is required to realign them. Notwithstanding these issues, this research clearly shows that full Stokes snapshot cameras have potential applications across a number of research fields. In addition to taking 3D images, they can also be used to characterize biological structures with full Stokes images [46]. Various other metasurface-based polarizers have been described, but few of them can operate at visible wavelengths, even after reductions in their period or size $[8,24,43,47-53]$.

Plasmonic nanostructures were previously introduced as promising candidates for light filtering applications [16,18-23,25-30,51,54]. Plasmonic behavior emerges in two basic situations [55]. Firstly, surface plasmon polaritons (SPP) occur if the plasmon is excited and propagated at the interface between the metal and dielectric with propagation constant: $\beta=\mathrm{k}_{0} \sqrt{\frac{\epsilon_{1} \epsilon_{2}}{\epsilon_{1}+\epsilon_{2}}}$, where $\mathrm{k}_{0}=\frac{\omega}{\mathrm{c}}=\frac{\frac{2 \pi c}{\lambda}}{\mathrm{c}}=\frac{2 \pi}{\lambda}, \epsilon_{1}$ and $\epsilon_{2}$ are the dielectric constants of the dielectric and metal. Secondly, localized surface plasmons (LSP) are evident when the excited plasmons propagate at the surface of the metallic nanoparticle (e.g., nanosphere, nanodisk, etc.), and are still coupled to the electromagnetic field. The behavior of the SPP case will depend largely on the materials chosen, whereas, for the LSP case, the peak wavelength mostly depends on the nanoparticle size [8,24,43,47-53].

Gold $(\mathrm{Au})$, silver $(\mathrm{Ag})$, and aluminum $(\mathrm{Al})$ are the generally preferred materials for plasmonic filters operating in the visible and NIR (VNIS) wavelengths (from $400 \mathrm{~nm}$ to $1000 \mathrm{~nm}$ ) because other metals, such as chromium (Cr), nickel (Ni), tungsten (W), and titanium (Ti), have larger absorption across the VNIS [55]. The resonant wavelength of $\mathrm{Au}$ is above $500 \mathrm{~nm}$, preventing its use in filters with target wavelengths less than this value. The strong oxidizing property of $\mathrm{Ag}$ also limits its application as its optical properties alter as it oxidizes. In addition, both $\mathrm{Au}$ and $\mathrm{Ag}$ require a seed layer (e.g., $\mathrm{Cr}, \mathrm{Ti}$ ) to improve their adhesion to silicon and this extra layer serves to reduce the transmission efficiency of the resulting optical filters [14]. Aluminum, as a CMOS compatible material, does not need a seed layer and is therefore more suitable for optical filtering applications that directly interface to a CMOS sensor $[16,21,29,30,54]$.

In this paper, we present our plasmonic DCPs based on a five-layer polarization sensitive nanostructure ( $\mathrm{Al}$ nanograting and nanocuboid combinations) with the capability to absorb circularly polarized light of an undesired direction. Our plasmonic polarizers use $\mathrm{Al}$ and $\mathrm{SiO}_{2}$ as the main materials, thus demonstrating good compatibility with CMOS image sensors, making them suitable for polarization imaging applications. Moreover, our DCPs have a wide operating band through the whole visible wavelengths and its CDT is largely insensitive to the angle of incidence between $0^{\circ}$ and $80^{\circ}$.

\section{Design and Simulations}

The overall structure of our dichroic circular polarizer (DCP), which is designed on a quartz substrate, is shown in Figure 1a. The first (top) layer of our DCP in Figure 1b is based on a 30-nm-thick $\mathrm{Al}$ nanograting array placed along the y-axis. The grating is $850 \mathrm{~nm}$ long and $30 \mathrm{~nm}$ wide, and is repeated along the x-direction with a period of $170 \mathrm{~nm}$. Figure 1c shows the second layer made of a 30-nm-thick nanocuboid array, each of them with a width of $30 \mathrm{~nm}$ and a length of $170 \mathrm{~nm}$. This layer was designed by rotating each nanocuboid $45^{\circ}$ clockwise around the $z$-axis. Aluminum nanogratings and nanocuboids are polarization sensitive, so that incident TE light will be suppressed when its electric field is perpendicular to the repeating nanogratings, or to the longer side of the nanocuboids. The overall metallic nanostructure in Figure $1 \mathrm{~g}$ is therefore created by continuing to rotate 
each successive layer by $45^{\circ}$ in the same clockwise direction around the $z$-axis, resulting in the structures shown in Figure 1d-f. Note that a 30-nm layer of $\mathrm{SiO}_{2}$ was deposited between each metallic nanograting/nanocuboid layer to act as isolation and the whole nanostructure was also encapsulated with $\mathrm{SiO}_{2}$.
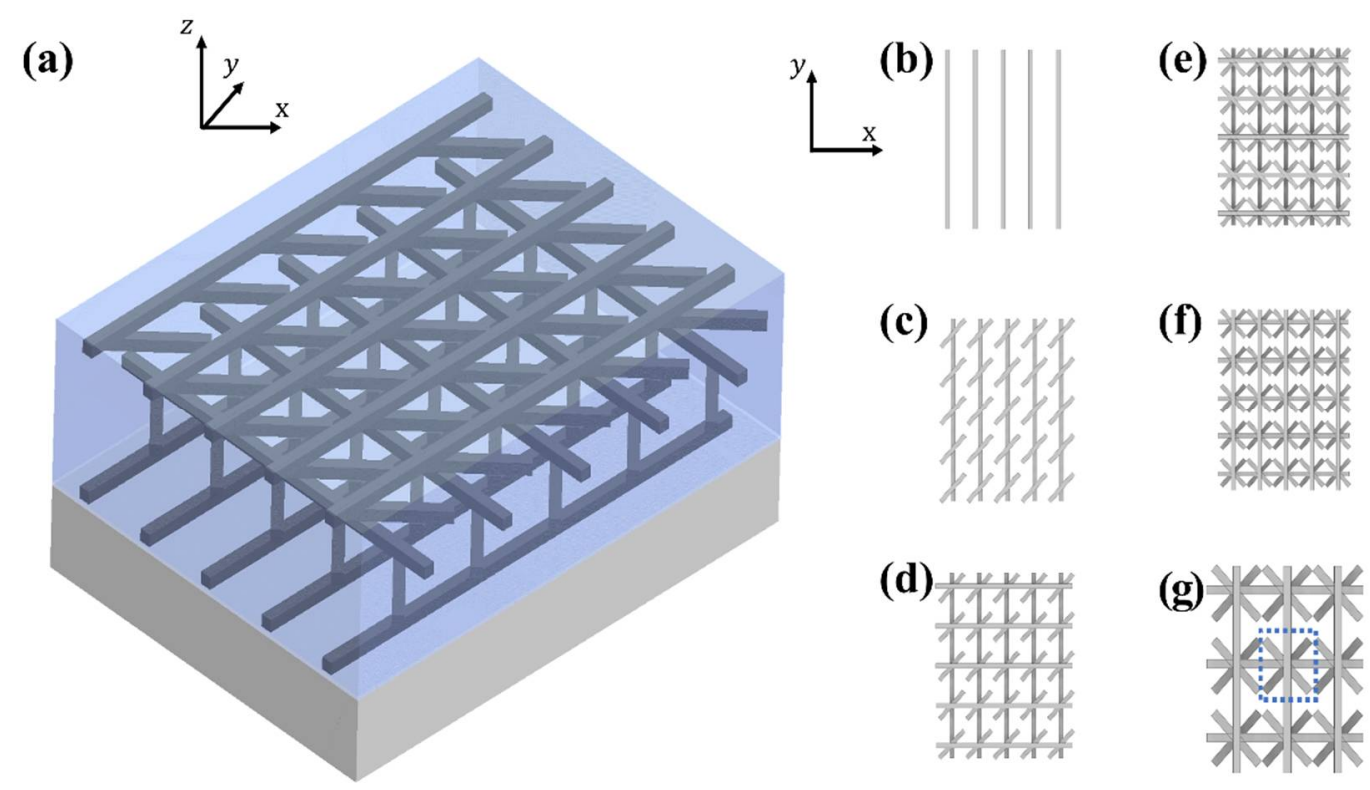

(c)

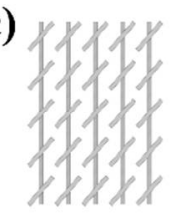

(f)

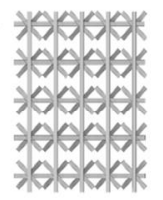

(d)

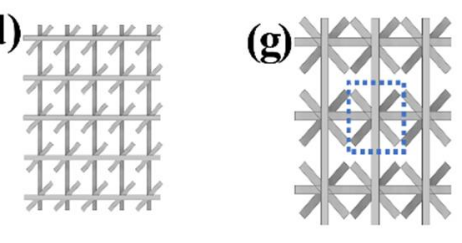

Figure 1. Nanostructures of the dichroic circular polarizer. (a) Overall structure in 3D view, showing the relative positions of the layers. Note: each layer is isolated with $30 \mathrm{~nm} \mathrm{SiO}_{2}$ (b) Top layer: Al nanograting aligned along the $y$-axis, (c) Second layer: Al nanocuboid array rotated $45^{\circ}$ around the $z$-axis, (d) Third layer: Al nanograting rotated by $45^{\circ}$ so it now aligns with the $x$-axis, (e) Fourth layer: Al nanocuboid array, again rotated $45^{\circ}$ around $z$-axis, (f) Fifth layer: Al nanograting rotated and aligned along the $y$-axis, (g) Top view of the dichroic circular polarizer with the simulation unit shown within the blue dashed square.

The simulations were performed using commercial software (COMSOL Multiphysics ${ }^{\circledR}$ ), applying finite element methods (FEMs). To reduce computation time, the small blue block in Figure $1 \mathrm{~g}$ was chosen as our simulation unit. Periodic boundary conditions (PBCs) were applied on all four sides and perfectly matched layers (PMLs) were used along the propagation direction, surrounded by scattering boundary conditions to absorb redundant light. Port boundaries were set between PMLs and their adjacent layers, and the LHP and RHP states were excited along the $\mathrm{Z}$ direction. The refractive indices of quartz substrate and isolation layers were 1.45 and the refractive index of $\mathrm{Al}$ was taken from Rakic's data [56]. Note that PBCs are replaced with PMLs when simulating a single pixel of the dichroic circular polarizer.

To compute the results, the transmission efficiency was calculated using the $\mathrm{S}$ parameter $\left|S_{21}\right|^{2}$ (in COMSOL the equation is abs (ewfd.S21^2)), and the CDT (circular dichroism transmission) was determined by the equation: CDT $=\frac{T_{1}-T_{2}}{T_{1}+T_{2}}$, where $T_{1}$ and $T_{2}$ are the transmission efficiencies of the two orthogonal polarization states, LHP and RHP, respectively. A higher CDT value means a better capability to detect and distinguish one direction of circularly polarized light from the other.

\section{Results}

The electric field shown in Figure 2 indicates the primary working principle for our left-handed DCP (LHDCP). Here, the LHP light is excited from the top. As the $\mathrm{Al}$ nanogratings/nanocuboids are polarization-sensitive, it is expected that the electric field will be more concentrated along the two sides in the y-direction than it is in the x-direction. Based on this concept, we built the model of Figure 1a. Figure 2a-e show the resulting electric field concentration seen from the top of the simulation unit of Figure 2f. As expected, most of the electric power is indeed concentrated on the two sides of the 
Al nanograting/nanocuboid along the diagonal direction. This behavior becomes much clearer as the LHP light propagates further into the structure (Figure 2e).

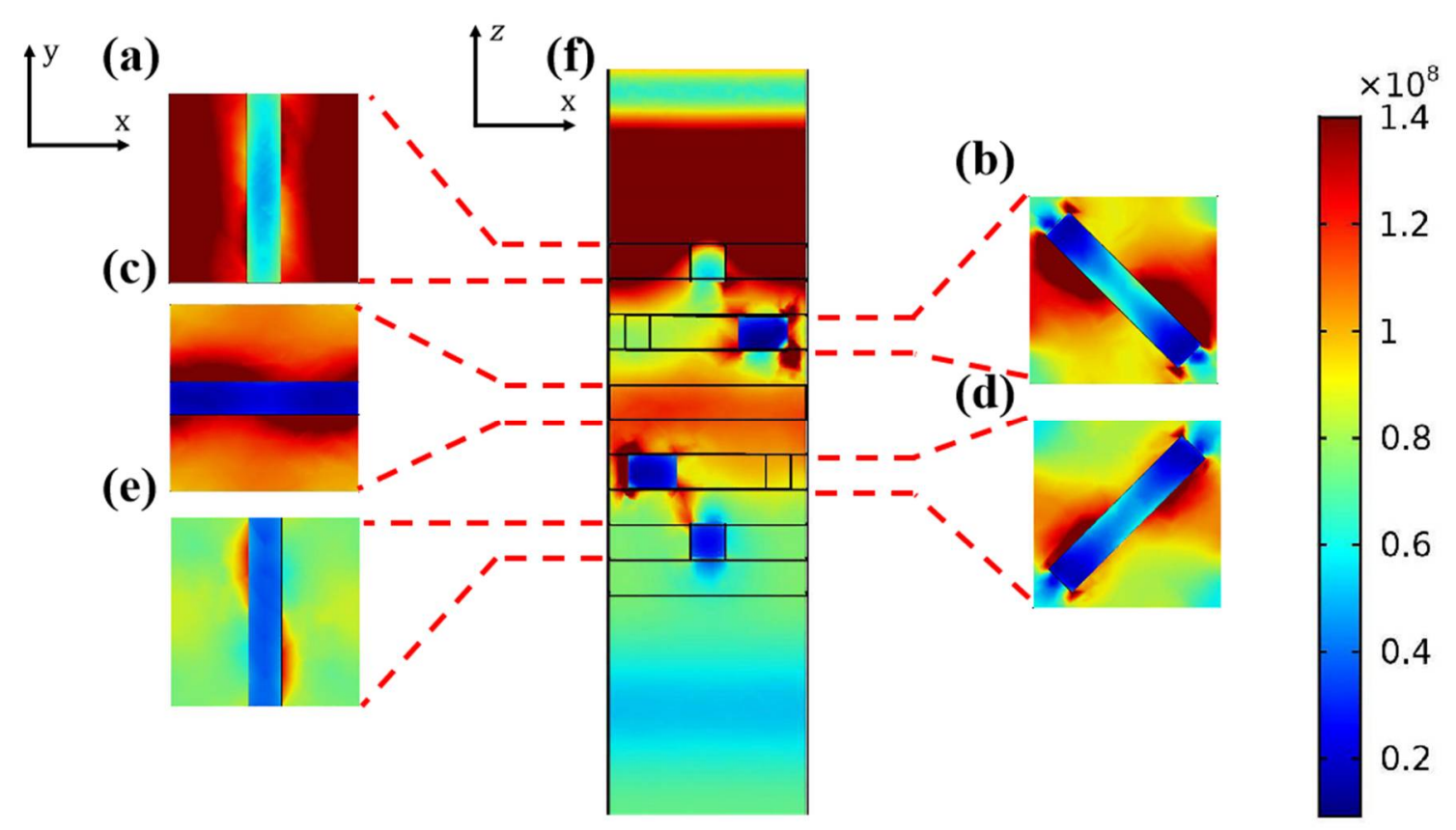

Figure 2. Electric field concentration of our proposed LHDCP for: (a) first (top) layer of Al nanograting aligned along the $y$-axis, (b) second layer of $\mathrm{Al}$ nanocuboids rotated $45^{\circ}$ around the $z$-axis, (c) third layer of $\mathrm{Al}$ nanograting rotated $45^{\circ}$ so it now aligns with the $x$-axis, (d) fourth layer of $\mathrm{Al}$ nanocuboid rotated around the $z$-axis by $45^{\circ}$, (e) fifth layer of $\mathrm{Al}$ nanograting rotated so it now aligns again with the $y$-axis, (f) Electric field cross-section of DCP model in the $x-z$ plane.

The transmission and absorption spectra in Figure 3, below, indicate the second working principle of our LHDCP under RHP light. From the graph, it can be seen that instead of reflecting RHP component, our LHDCP absorbs and therefore filters out RHP light. In contrast, the LHP light passes through the filter. As a result, problems such as interference between the incident and reflected light will be virtually eliminated.

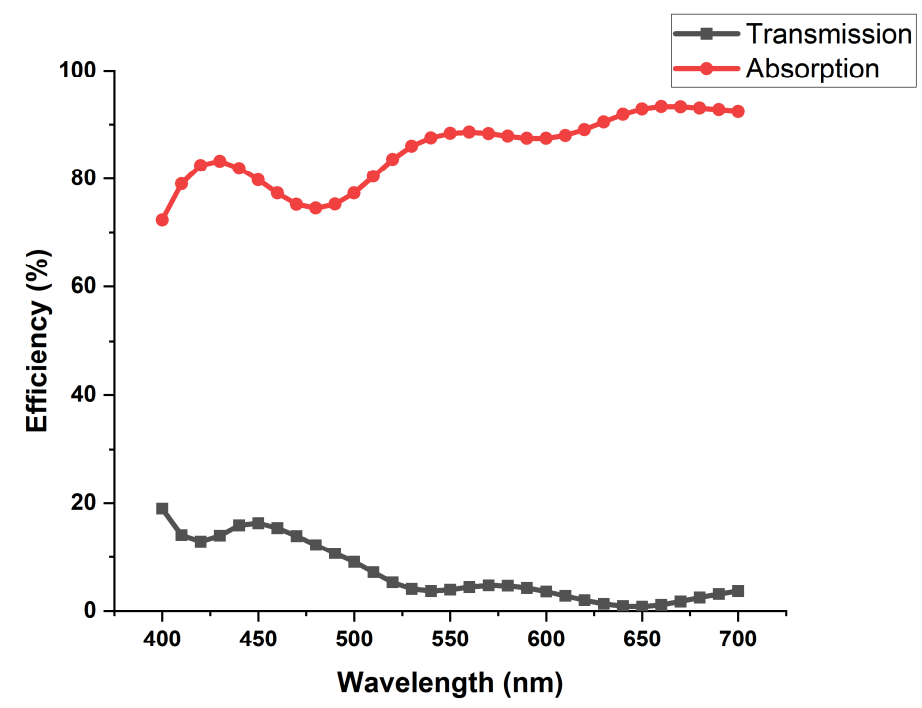

Figure 3. Black and red lines are the transmission and absorption spectra of our LHDCP under RHP incident light.

As mentioned previously, nanogratings and nanocuboids are sensitive to TM polarized light so that, when the nano-structures are rotated through an angle, the polarization angles 
of incident TM light change correspondingly. Moving the rotated structures to a different layer will introduce a phase difference. Therefore, LHP and RHP light can be distinguished by adjusting the layer components. Figure 4 describes our investigation between onelayer structure and five-layer structures. Note that the first, third, and fifth layers are nanogratings, whereas the second and fourth are the nanocuboids, previously shown in Figure 1. It can be seen from Figure 4 that, when we increase the number of rotations or layers, the CDT becomes larger and the wavelength range where the CDT greater than 0.5 becomes wider.

(a)

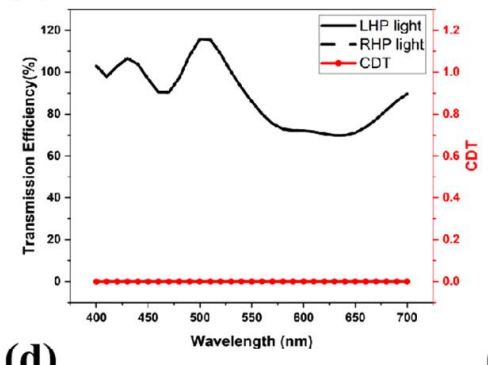

(d)

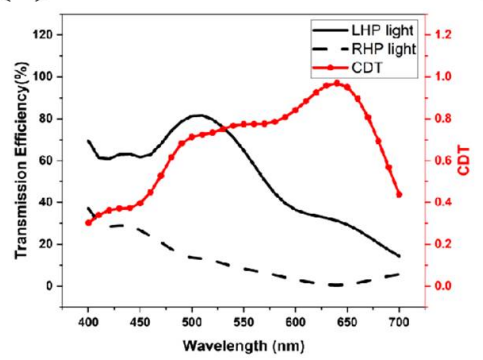

(b)

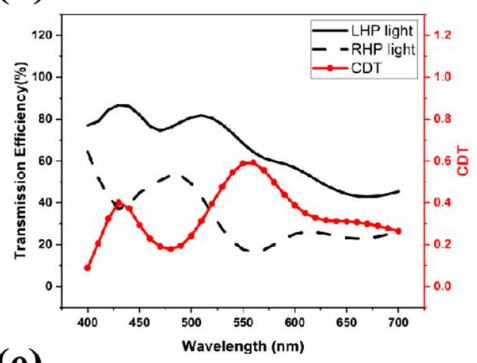

(e)

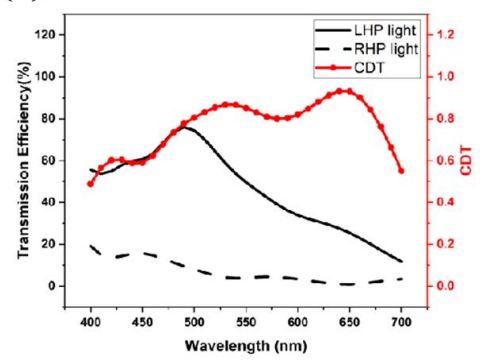

(c)

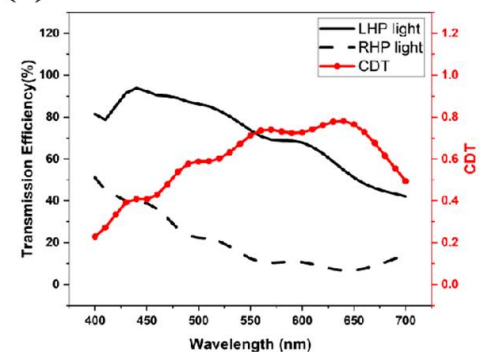

Figure 4. The transmission efficiency of LHP, RHP light and CDT of our proposed nanostructures, the combinations of nanogratings and nanocuboids for: (a) 1-layer, (b) 2-layer, (c) 3-layer, (d) 4-layer, (e) 5-layer.

The reasoning behind this combination of nanogratings and nanocuboids is as follows. The function of a plasmonic nanograting is based on surface plasmon polaritons (SPP), and it is well known that any metallic nanostructures based on SPP will be sensitive to the angle of incidence. In contrast, the behavior of a nanocuboid surface depends on localized surface polaritons (LSP), which are insensitive to the angle of incidence. As shown in Figure 5, circular polarizers based only on Al nanocuboid arrays cannot achieve a wide operating spectrum across visible wavelengths whereas combinations of nanograting and nanocuboid surfaces are able to both function over a wide bandwidth in the visible and be insensitive to the angle of incidence.

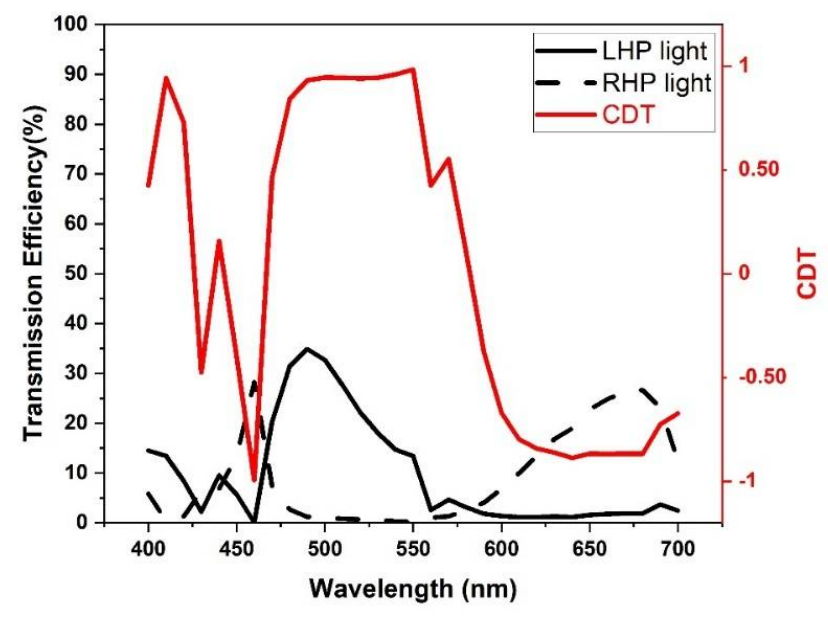

Figure 5. The transmission efficiency of LHP, RHP light and CDT of 5-layer nanocuboids. 
Based on the principles of the LHDCP outlined above, we optimized the size of our DCPs by varying the width and period parameters of our nanostructures over a narrow range centered on $30 \mathrm{~nm}$ and $170 \mathrm{~nm}$, respectively. The final result is shown in Figure 6a and the optimization process is the following: The period was first changed from $170 \mathrm{~nm}$ to $160 \mathrm{~nm}$ (Figure 6b) and $180 \mathrm{~nm}$ (Figure 6c) while maintaining the width at $30 \mathrm{~nm}$. Then, the width was set in turn to $20 \mathrm{~nm}$ (Figure 6d) and $40 \mathrm{~nm}$ (Figure 6e) while maintaining the period at $170 \mathrm{~nm}$. It can be seen that, as the period increases, the range of wavelengths over which the transmission efficiency of the LHP light is larger than $50 \%$ also increases. At the same time, the range where the CDT is larger than 0.5 decreases. On the other hand, increasing the width results in a wider range where the CDT is larger than 0.5 , but narrows the transmission efficiency range. Therefore, after evaluating the results in Figure 6a, our optimized width and period parameter were set at their original values of $30 \mathrm{~nm}$ and $170 \mathrm{~nm}$, respectively.

(a)

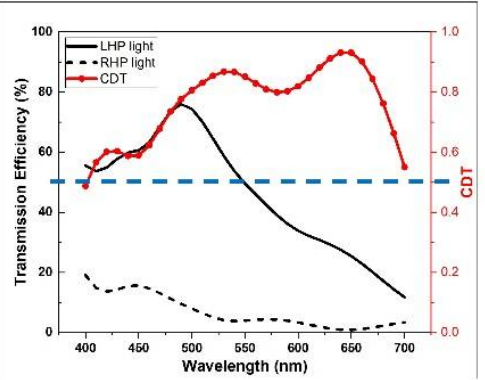

(b)

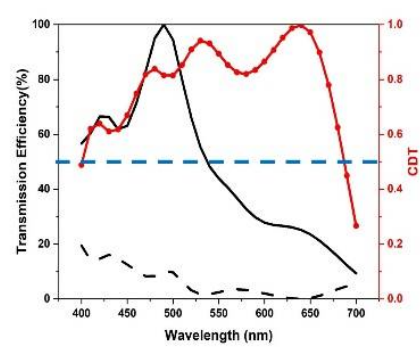

(d)

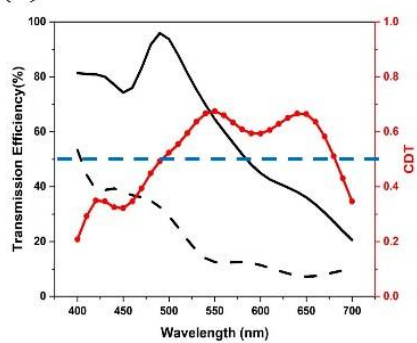

(c)

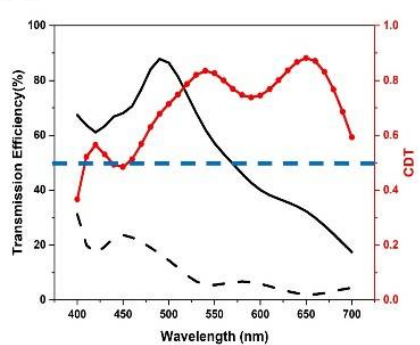

(e)

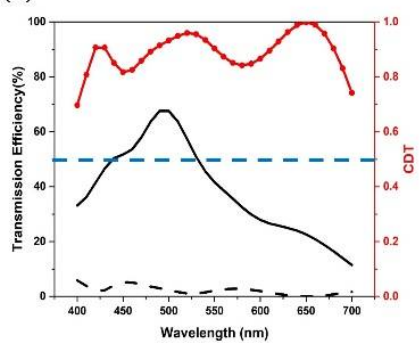

Figure 6. Black lines are the transmission spectra of our LHDCP under LHP and RHP incident light. The red line is the CDT calculated from the above two transmission spectra. Results for: (a) Period 170 nm, Width 30 nm, (b) Period 160 nm, Width 30 nm, (c) Period 180 nm, Width 30 nm, (d) Period 170 nm, Width 20 nm, (e) Period 170 nm, Width 40 nm.

The resulting transmission spectra of the left circular polarizer under LHP and RHP incident light and CDT are shown in Figure 6a. The solid black line is the transmission spectrum of LHDCP when LHP light is incident, which has a maximum transmission efficiency of $78 \%$ at $500 \mathrm{~nm}$. The black line is the transmission spectrum of LHDCP under RHP incident light. Although the transmission efficiency decreases when the wavelength is tuned to the red range, the CDT reaches more than 0.9 at $650 \mathrm{~nm}$, and the extinction ratio is 30. The polarizer filters out almost all the RHP light. Therefore, from the CDT results in Figure 6a, our LHDCP is sufficient for polarized imaging applications. The lower transmission efficiency at some wavelengths can be compensated by increasing the exposure time of the image sensor.

Our LHDCP also exhibits good performance over a wide range of incident angles. We varied the incident angles for both LHP and RHP light show in Figure 7 and calculated the corresponding CDT. Figure 8 shows the CDT lines under incident light with angles up to $80^{\circ}$. As can be seen, the CDT is almost constant with angles over the visible range, indicating that our DCP has a large tolerance to incident angles and will not require a lens with a particular $f$-number to support polarized imaging. Therefore, it is likely to be suitable for a wide range of polarized imaging applications. 


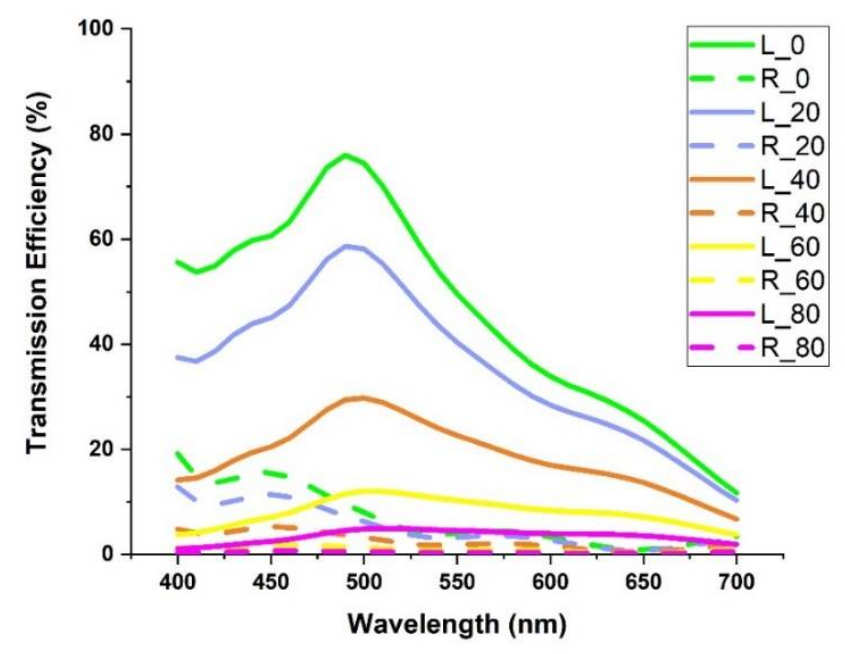

Figure 7. The transmission efficiency of LHDCP with incident LHP and RHP light vs. angle of incidence, varied in $20^{\circ}$ steps from $0^{\circ}$ to $80^{\circ}$. Solid lines are LHP and dotted lines are RHP.

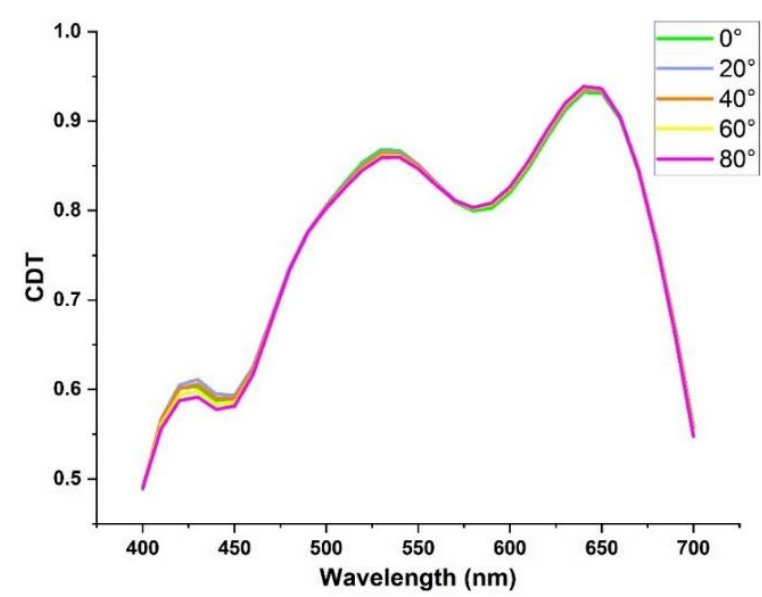

Figure 8. CDT under incident light with different angles from $0^{\circ}$ to $80^{\circ}$.

In order to evaluate the operation of our polarizer in a real camera system, we undertook a number of simulations in which a small array was size-matched to a commercial image sensor. Due to its underlying plasmonic nature. There is a trade-off between the optical properties (CDT and transmission efficiency) of our DCP and its size. Reducing the size of plasmonic DCP will degrade its optical properties as each block will encompass fewer nanoparticle units. Therefore, we considered a CMOS image sensor with a pixel size around 2.4 um (e.g., a Sony CMOS Exmor sensor (IMX183) with 20-M resolution). To demonstrate this issue and to obtain the transmission efficiency and CDT of our LHDCP when interfaced to this image sensor, we replaced the PBCs with PMLs and used an array of $15 \times 15$ elements, each $2.4 \mu \mathrm{m} \times 2.4 \mu \mathrm{m}$ in size. The simulation results are shown in Figure 9. The transmission efficiency is lower than that of the previous periodic models ( $<60 \%$ vs. a peak around $75 \%$ ) due to the reduced number of nano-scale elements within the smaller overall area. The CDT reaches 0.5 at a lower wavelength (approximately $660 \mathrm{~nm}$ vs. $750 \mathrm{~nm}$ ) for the same reason. Note that reducing the number of elements will reduce the peak CDT so it rolls off at a wavelength lower than $700 \mathrm{~nm}$. This implies that $700 \mathrm{~nm}$ is more-or-less a hard limit for our LHDCP — it will not operate on polarized light at longer wavelengths but will simply act as a phase shifter in this region. However, its performance at visible wavelengths is still better than comparable DCP systems. Therefore, we think that our DCP is suitable for use as an image sensor with pixel sizes larger than $2.4 \mu \mathrm{m}$. This problem can also be addressed by trading off size against image resolution, and by arranging each DCP to cover more pixels-for example, a small block of $2 \times 2$ each. 


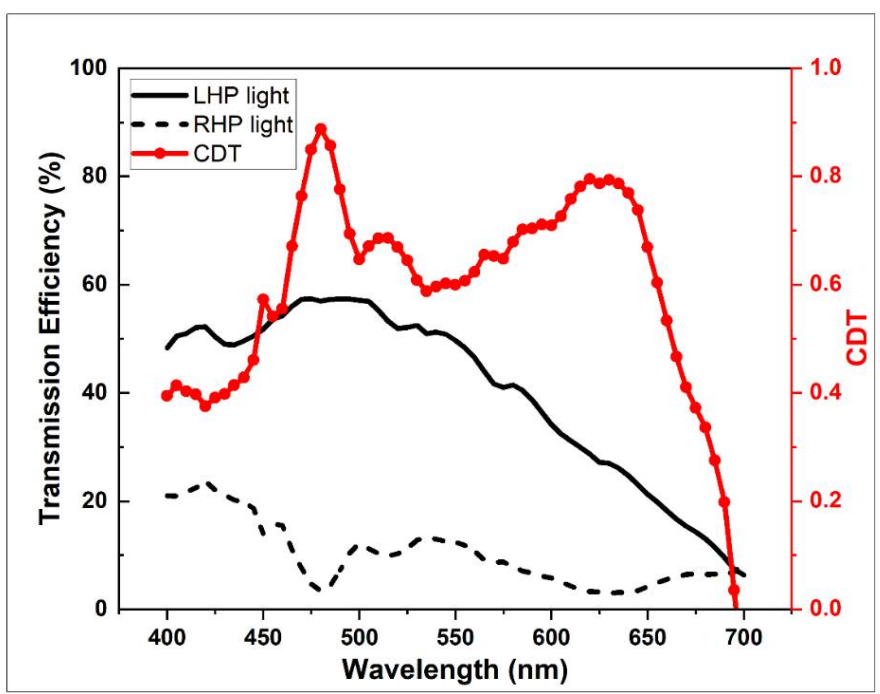

Figure 9. Simulation results of smaller LHDCP in a single-pixel size of $2.4 \times 2.4 \mu \mathrm{m}^{2}$ : Black lines are the transmission spectra of our LHDCP under LHP and RHP incident light, the red line is the CDT calculated from the above two transmission spectra.

Being able to determine intensity values for the RHP and LHP components means it becomes possible to calculate the fourth Stokes parameter $S_{3}$, which is equivalent to the difference between the circular Jones vectors, $E$, so that $S_{3} \equiv\left\langle E_{r}^{2}\right\rangle-\left\langle E_{l}^{2}\right\rangle$, where the subscripts identify the right and left circular bases. By integrating our DCPs with linear polarizers on the image sensor, we can create a full Stokes polarization camera. Note that the linear polarizers can be achieved with $\mathrm{Al}$ nanogratings, which is a well-developed technology in the market. A full Stokes polarization camera with our DCPs would have a broad operating range across the visible spectrum and would be able to measure the Stokes parameters and position these on the Poincaré sphere [44], something that would be very useful in bio-inspired designs that mimic insect vision [45].

\section{Conclusions}

We have developed a dichroic circular polarizer (DCP) based on plasmonic nanostructures. The DCP comprises five layers of Al nanograting/nanocuboid surfaces embedded in $\mathrm{SiO}_{2}$ built onto a quartz substrate. The whole layered structure comprises only around a hundred nanometer of CMOS compatible dielectric and metal materials. Thus, this kind of DCP is not bulky and has lower optical loss that traditional DCP systems. The CDT of the device can reach more than 0.9 with maximum transmission efficiencies larger than $78 \%$ at visible wavelengths. We also demonstrate that our DCP is insensitive to angle of incidence by calculating its CDT values under varying angles up to $80^{\circ}$. During device integration, the operating wavelengths, transmission efficiency, and CDT can be simply tuned by varying the size, spacing, and period of the plasmonic nanostructures. Thus, the integration of these circular polarizers on a photodetector array is less complicated and much cheaper than previous methods. In addition, the CDT is still high enough to be used when the structure size shrinks to $2.4 \mu \mathrm{m}$, showing its capability and compatibility with most current image sensors for polarization imaging applications.

Author Contributions: Conceptualization, X.H. (Xin He) and X.H. (Xiang Hao); Data curation, J.Z. and X.H. (Xin He); Formal analysis, J.Z. and X.H. (Xin He); Funding acquisition, X.L. and X.H. (Xiang Hao); Investigation, J.Z., X.H. (Xin He) and X.H. (Xiang Hao); Methodology, J.Z. and X.H. (Xin He); Project administration, X.L. and X.H. (Xiang Hao); Resources, X.H. (Xin He), P.B., X.S., Z.C., W.Z., X.L. and X.H. (Xiang Hao); Software, J.Z., X.H. (Xin He), X.S., Z.C. and W.Z.; Supervision, X.H. (Xin He) and X.H. (Xiang Hao); Validation, J.Z. and X.H. (Xin He); Visualization, J.Z., X.H. (Xin He), X.S., Z.C. and W.Z.; Writing—original draft, J.Z., X.H. (Xin He), P.B. and X.H. (Xiang Hao); Writing-review \& 
editing, J.Z., X.H. (Xin He), P.B., X.S., Z.C., W.Z., X.L. and X.H. (Xiang Hao). All authors have read and agreed to the published version of the manuscript.

Funding: This work was financially supported by the Major Research Plan of the National Natural Science Foundation of China (92050115) and Zhejiang Provincial Natural Science Foundation of China (LZ21F050003).

Data Availability Statement: The data presented in this study are available on request from the corresponding author.

Conflicts of Interest: The authors declare no conflict of interest in this work.

\section{References}

1. John-Herpin, A.; Kavungal, D.; von Mücke, L.; Altug, H. Infrared Metasurface Augmented by Deep Learning for Monitoring Dynamics between All Major Classes of Biomolecules. Adv. Mater. 2021, 33, 2006054. [CrossRef]

2. Shaw, T.; Mitra, D. Metasurface-based radiative near-field wireless power transfer system for implantable medical devices. IET Microw. Antennas Propag. 2019, 13, 1974-1982. [CrossRef]

3. Tittl, A.; Leitis, A.; Liu, M.; Yesilkoy, F.; Choi, D.-Y.; Neshev, D.N.; Kivshar, Y.S.; Altug, H. Imaging-based molecular barcoding with pixelated dielectric metasurfaces. Science 2018, 360, 1105-1109. [CrossRef] [PubMed]

4. Zhou, J.; Liu, S.; Qian, H.; Li, Y.; Luo, H.; Wen, S.; Zhou, Z.; Guo, G.; Shi, B.; Liu, Z. Metasurface enabled quantum edge detection. Sci. Adv. 2020, 6, eabc4385. [CrossRef]

5. Huang, Y.; Xie, X.; Pu, M.; Guo, Y.; Xu, M.; Ma, X.; Li, X.; Luo, X. Dual-Functional Metasurface toward Giant Linear and Circular Dichroism. Adv. Opt. Mater. 2020, 8, 1902061. [CrossRef]

6. Wang, S.; Deng, Z.-L.; Wang, Y.; Zhou, Q.; Wang, X.; Cao, Y.; Guan, B.-O.; Xiao, S.; Li, X. Arbitrary polarization conversion dichroism metasurfaces for all-in-one full Poincaré sphere polarizers. Light Sci. Appl. 2021, 10, 24. [CrossRef]

7. Berndt, A.J.; Hwang, J.; Islam, M.D.; Sihn, A.; Urbas, A.M.; Ku, Z.; Lee, S.J.; Czaplewski, D.A.; Dong, M.; Shao, Q.; et al Poly(sulfur-random-(1,3-diisopropenylbenzene)) based mid-wavelength infrared polarizer: Optical property experimental and theoretical analysis. Polymer 2019, 176, 118-126. [CrossRef]

8. Mun, S.-E.; Hong, J.; Yun, J.-G.; Lee, B. Broadband circular polarizer for randomly polarized light in few-layer metasurface. Sci. Rep. 2019, 9, 2543. [CrossRef]

9. Circular Polarizers. Available online: https://www.edmundoptics.com/f/circular-polarizers-cp42he-and-cp42her/14781/ (accessed on 19 June 2021).

10. Polymer Circular Polarizer. Available online: https://www.thorlabs.com/newgrouppage9.cfm?objectgroup_id=10498 (accessed on 19 June 2021).

11. Myhre, G.; Sayyad, A.; Pau, S. Patterned color liquid crystal polymer polarizers. Opt. Express 2010, 18, 27777-27786. [CrossRef]

12. Miyagi, K.; Teramoto, Y. Elucidation of the Mechanism of Stress-Induced Circular Dichroic Inversion of Cellulosic/Polymer Liquid Crystalline Composites. Macromolecules 2020, 53, 3250-3254. [CrossRef]

13. Park, G.; Choi, Y.-S.; Yun, H.S.; Yoon, D.K. Fabrication of Bilayer Dichroic Films Using Liquid Crystal Materials for Multiplex Applications. ACS Appl. Mater. Interfaces 2020, 12, 45315-45321. [CrossRef] [PubMed]

14. Knight, M.W.; King, N.S.; Liu, L.; Everitt, H.O.; Nordlander, P.; Halas, N.J. Aluminum for plasmonics. ACS Nano 2014, 8, 834-840. [CrossRef] [PubMed]

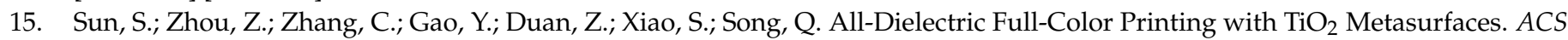
Nano 2017, 11, 4445-4452. [CrossRef] [PubMed]

16. He, X.; O'Keefe, N.; Liu, Y.; Sun, D.; Uddin, H.; Nirmalathas, A.; Unnithan, R.R. Transmission enhancement in coaxial hole array based plasmonic color filter for image sensor applications. IEEE Photonics J. 2018, 10, 1-9. [CrossRef]

17. Koirala, I.; Lee, S.-S.; Choi, D.-Y. Highly transmissive subtractive color filters based on an all-dielectric metasurface incorporating $\mathrm{TiO}_{2}$ nanopillars. Opt. Express 2018, 26, 18320-18330. [CrossRef]

18. Chen, Q.; Cumming, D.R. High transmission and low color cross-talk plasmonic color filters using triangular-lattice hole arrays in aluminum films. Opt. Express 2010, 18, 14056-14062. [CrossRef]

19. Xu, T.; Wu, Y.-K.; Luo, X.; Guo, L.J. Plasmonic nanoresonators for high-resolution colour filtering and spectral imaging. Nat. Commun. 2010, 1, 1-5. [CrossRef] [PubMed]

20. Yokogawa, S.; Burgos, S.P.; Atwater, H.A. Plasmonic color filters for CMOS image sensor applications. Nano Lett. 2012, 12, 4349-4354. [CrossRef]

21. Burgos, S.P.; Yokogawa, S.; Atwater, H.A. Color imaging via nearest neighbor hole coupling in plasmonic color filters integrated onto a complementary metal-oxide semiconductor image sensor. ACS Nano 2013, 7, 10038-10047. [CrossRef]

22. Do, Y.S.; Park, J.H.; Hwang, B.Y.; Lee, S.M.; Ju, B.K.; Choi, K.C. Plasmonic color filter and its fabrication for large-area applications. Adv. Opt. Mater. 2013, 1, 133-138. [CrossRef]

23. Honma, H.; Takahashi, K.; Fukuhara, M.; Ishida, M.; Sawada, K. Free-standing aluminium nanowire arrays for high-transmission plasmonic colour filters. Micro Nano Lett. 2014, 9, 891-895. [CrossRef] 
24. Jia, S.; Wan, X.; Bao, D.; Zhao, Y.J.; Cui, T.J. Independent controls of orthogonally polarized transmitted waves using a Huygens metasurface. Laser Photonics Rev. 2015, 9, 545-553. [CrossRef]

25. McCrindle, I.J.H.; Grant, J.P.; Gouveia, L.C.P.; Cumming, D.R.S. Infrared plasmonic filters integrated with an optical and terahertz multi-spectral material. Phys. Status Solidi 2015, 212, 1625-1633. [CrossRef]

26. Ema, D.; Kanamori, Y.; Sai, H.; Hane, K. Plasmonic color filters integrated on a photodiode array. Electron. Commun. Jpn. 2018, 101, 95-104. [CrossRef]

27. Fleischman, D.; Fountaine, K.T.; Bukowsky, C.R.; Tagliabue, G.; Sweatlock, L.A.; Atwater, H.A. High spectral resolution plasmonic color filters with subwavelength dimensions. Acs Photonics 2019, 6, 332-338. [CrossRef]

28. Panchenko, E.; Wesemann, L.; Gómez, D.E.; James, T.D.; Davis, T.J.; Roberts, A. Ultracompact camera pixel with integrated plasmonic color filters. Adv. Opt. Mater. 2019, 7, 1900893. [CrossRef]

29. He, X.; Liu, Y.; Beckett, P.; Uddin, M.H.; Nirmalathas, A.; Unnithan, R.R. Hybrid Color Filters for Multispectral Imaging. Adv. Theory Simul. 2020, 3, 2000137. [CrossRef]

30. He, X.; Liu, Y.; Beckett, P.; Uddin, H.; Nirmalathas, A.; Unnithan, R.R. CMY camera using a nanorod filter mosaic integrated on a CMOS image sensor. OSA Contin. 2021, 4, 229-238. [CrossRef]

31. Zhao, Y.; Belkin, M.A.; Alù, A. Twisted optical metamaterials for planarized ultrathin broadband circular polarizers. Nat. Commun. 2012, 3, 870. [CrossRef] [PubMed]

32. Zeng, B.; Gao, Y.; Bartoli, F.J. Ultrathin Nanostructured Metals for Highly Transmissive Plasmonic Subtractive Color Filters. Sci. Rep. 2013, 3, 2840. [CrossRef] [PubMed]

33. Polarization Camera with 5.0 MP Monochrome CMOS Sensor. Available online: https:/ /www.thorlabs.com/newgrouppage9 .cfm?objectgroup_id=13033 (accessed on 19 June 2021).

34. Hofmeister, H.; Drost, W.G.; Berger, A. Oriented prolate silver particles in glass-Characteristics of novel dichroic polarizers. Nanostructured Mater. 1999, 12, 207-210. [CrossRef]

35. Moiseev, S.G. Thin-film polarizer made of heterogeneous medium with uniformly oriented silver nanoparticles. Appl. Phys. A 2011, 103, 775-777. [CrossRef]

36. Muravitskaya, A.; Movsesyan, A.; Kostcheev, S.; Adam, P.-M. Polarization switching between parallel and orthogonal collective resonances in arrays of metal nanoparticles. J. Opt. Soc. Am. B 2019, 36, E65-E70. [CrossRef]

37. Glukhov, I.A.; Dadoenkova, Y.S.; Bentivegna, F.F.L.; Moiseev, S.G. Deterministic aperiodic photonic crystal with a $2 \mathrm{D}$ array of metallic nanoparticles as polarization-sensitive dichroic filter. J. Appl. Phys. 2020, 128, 053101. [CrossRef]

38. Li, Z.; Liu, W.; Cheng, H.; Chen, S.; Tian, J. Spin-Selective Transmission and Devisable Chirality in Two-Layer Metasurfaces. Sci. Rep. 2017, 7, 8204. [CrossRef] [PubMed]

39. Gansel, J.K.; Thiel, M.; Rill, M.S.; Decker, M.; Bade, K.; Saile, V.; von Freymann, G.; Linden, S.; Wegener, M. Gold Helix Photonic Metamaterial as Broadband Circular Polarizer. Science 2009, 325, 1513-1515. [CrossRef]

40. Ji, R.; Wang, S.-W.; Liu, X.; Chen, X.; Lu, W. Broadband circular polarizers constructed using helix-like chiral metamaterials. Nanoscale 2016, 8, 14725-14729. [CrossRef]

41. Yu, Y.; Yang, Z.; Li, S.; Zhao, M. Higher extinction ratio circular polarizers with hetero-structured double-helical metamaterials. Opt. Express 2011, 19, 10886-10894. [CrossRef]

42. Nanoscribe Quantum X, Nanoscribe GmbH \& Co. Available online: https:/ /www.nanoscribe.com/en/products/quantum-X (accessed on 19 June 2021).

43. Chen, Y.; Gao, J.; Yang, X. Direction-Controlled Bifunctional Metasurface Polarizers. Laser Photonics Rev. 2018, 12, 1800198. [CrossRef]

44. Kwon, D.-H.; Werner, P.L.; Werner, D.H. Optical planar chiral metamaterial designs for strong circular dichroism and polarization rotation. Opt. Express 2008, 16, 11802-11807. [CrossRef]

45. Rubin, N.A.; D'Aversa, G.; Chevalier, P.; Shi, Z.; Chen, W.T.; Capasso, F. Matrix Fourier optics enables a compact full-Stokes polarization camera. Science 2019, 365, eaax1839. [CrossRef] [PubMed]

46. Otani, Y. Snapshot Full Stokes Imager by Polarization Cameras and Its Application to Bio-Imaging. Proc. SPIE 2021, $11709,1170904$.

47. Bai, J.; Wang, C.; Chen, X.; Basiri, A.; Wang, C.; Yao, Y. Chip-integrated plasmonic flat optics for mid-infrared full-Stokes polarization detection. Photonics Res. 2019, 7, 1051-1060. [CrossRef]

48. Kang, L.; Wang, C.-Y.; Guo, X.; Ni, X.; Liu, Z.; Werner, D.H. Nonlinear Chiral Meta-Mirrors: Enabling Technology for Ultrafast Switching of Light Polarization. Nano Lett. 2020, 20, 2047-2055. [CrossRef] [PubMed]

49. Kou, W.; Zhang, Y.; Chen, T.; Yang, Z.; Liang, S. Multifunctional linear-polarized terahertz focusing metasurface. Microw. Opt. Technol. Lett. 2020, 62, 2721-2727. [CrossRef]

50. Sheersha, J.A.; Nasimuddin, N.; Alphones, A. A high gain wideband circularly polarized antenna with asymmetric metasurface. Int. J. RF Microw. Comput. Aided Eng. 2019, 29, e21740. [CrossRef]

51. Song, H.; Ma, Y.; Han, Y.; Shen, W.; Zhang, W.; Li, Y.; Liu, X.; Peng, Y.; Hao, X. Deep-Learned Broadband Encoding Stochastic Filters for Computational Spectroscopic Instruments. Adv. Theory Simul. 2021, 4, 2000299. [CrossRef]

52. Swain, R.; Mishra, R.K. Metasurface cavity antenna for broadband high-gain circularly polarized radiation. Int. J. RF Microw. Comput. Aided Eng. 2019, 29, e21609. [CrossRef]

53. Ta, S.X.; Nguyen, M.; Nguyen, T.; Nguyen, T.K.; Nguyen Khac, K.; Dao-Ngoc, C. Low-profile broadband dual-polarized antenna utilizing metasurface. Microw. Opt. Technol. Lett. 2018, 60, 2534-2539. 
54. He, X.; Liu, Y.; Ganesan, K.; Ahnood, A.; Beckett, P.; Eftekhari, F.; Smith, D.; Uddin, M.H.; Skafidas, E.; Nirmalathas, A. A single sensor based multispectral imaging camera using a narrow spectral band color mosaic integrated on the monochrome CMOS image sensor. APL Photonics 2020, 5, 046104. [CrossRef]

55. Ding, F.; Yang, Y.; Deshpande, R.A.; Bozhevolnyi, S.I. A review of gap-surface plasmon metasurfaces: Fundamentals and applications. Nanophotonics 2018, 7, 1129-1156. [CrossRef]

56. Rakić, A.D. Algorithm for the determination of intrinsic optical constants of metal films: Application to aluminum. Appl. Opt. 1995, 34, 4755-4767. [CrossRef] [PubMed] 